2006 ERSD Annual Report

DOE-BER Environmental Remediation Sciences Project \#1024840

\title{
Stability of U(VI)- and Tc(VII) reducing microbial communities to environmental perturbation: a thermodynamic network model and intermediate-scale experiments
}

\author{
PIs: James P. McKinley ${ }^{1}$ \\ Co-PIs: Chongxuan Liu ${ }^{1}$ Jack Istok $^{2}$, Lee Krumholz ${ }^{3}$ \\ ${ }^{1}$ Pacific Northwest National Laboratory, Richland, WA \\ ${ }^{2}$ Oregon State University, Corvallis, OR \\ ${ }^{3}$ University of Oklahoma, Norman, OK
}

\section{Research Objectives:}

The project is a collaborative task with a larger project headed by Jack Istok at Oregon State University, which is conducted under the same title. The project was conceptualized as follows. A 'geochemical' model of microbial communities was hypothesized, in which microbes were characterized as mineral species according to the chemical transformations they used for metabolic function. The iron-reducing bacteria, for example, would be represented by the ironreducing chemical reaction, including a specific electron donor, the fraction of the consumed donor used for biomass maintenance or growth, and a free energy for the reaction. The pseudomineral species would then be included in a standard geochemical model, and community succession could be calculated according to the thermodynamically favored microbially mediated reactions under progressive consumption of electron donors and receptors, and evolving geochemical conditions. The project includes relatively minor participation by the University of Oklahoma and Pacific Northwest National Laboratory, with the major component at OSU. The PNNL project was funded to provide assistance to Dr. Istok in formulating the appropriate modeling approach and geochemical constraints on the modeling effort. The objectives we have pursued are

- To evaluate and recommend a standard geochemical modeling code amenable to modification for modeling microbial communities

- Provide geochemical expertise in bounding the modeling effort for relevance to geochemical systems, and guidance in approaches to geochemical modeling.

\section{Research Progress and Implications:}

We supported the OSU effort to choose an appropriate modeling package. The University of Illinois software package 'Geochemists Work Bench', vended by Rockware, Inc., was chosen and adopted for this effort. The software was coupled to a MatLab preprocessor constructed with our assistance by OSU. We provided assistance in choosing relevant geochemical approaches to representing microbial metabolic transformations in the subsurface, and continue 
to provide advice and assistance during the construction and implementation of the microbial community model.

Most of the activities described occurred in the first year of the project, which is beginning its third and final year. Figure 1 is a modeling result based on conditions at the Rifle, Colorado, UMTRA site, and shows a hypothetical progression of the subsurface during acetate amendment and mineralization. Microbial community reactions and community dominance were computed to progress from acetate mineralization by aerobic reaction through reactions involving the reduction of nitrate, sulfate, manganese, and iron. Eventually, with electron donors depleted, the system was computed to reach a point of methanogenesis. Progressions such as this have been hypothesized and observed in nature.

Our activities have been relatively small in FY 2006, due to the need for OSU personnel to build and test a comprehensive thermodynamic database of microbial species, and to refine the MatLab pre-modeling protocol. We have provided advice on the thermodynamic treatment of particular chemical species, the relevance of particular mineral and chemical species, and suggested approaches to modeling some microbial community members. We have built up significant carry over funds from our modest $\$ 80 \mathrm{~K}$ per year budget, and have begun planning with OSU for a series of model-testing experimental activities to be conducted in FY 2007.

\section{Planned Activities:}

The project is at a point where modeling of experimental or in situ microbial communities could be attempted. We are collaborating with Drs. Istok and Krumholz to plan and execute such experiments in FY 2007, to provide a test of this modeling approach. These activities will exhaust our remaining carry over funds and the FY 2007 allocation. 
Figure 1. Simulated response of in-situ microbial community to acetate amendment. The horizontal scale represents moles of acetate reacted. The results depict a community succession from dominant aerobes to progressively less efficient energy extraction by nitrate, sulface, manganese, and iron reducing communities. When electron accepting compounds are depleted methanogens dominate.

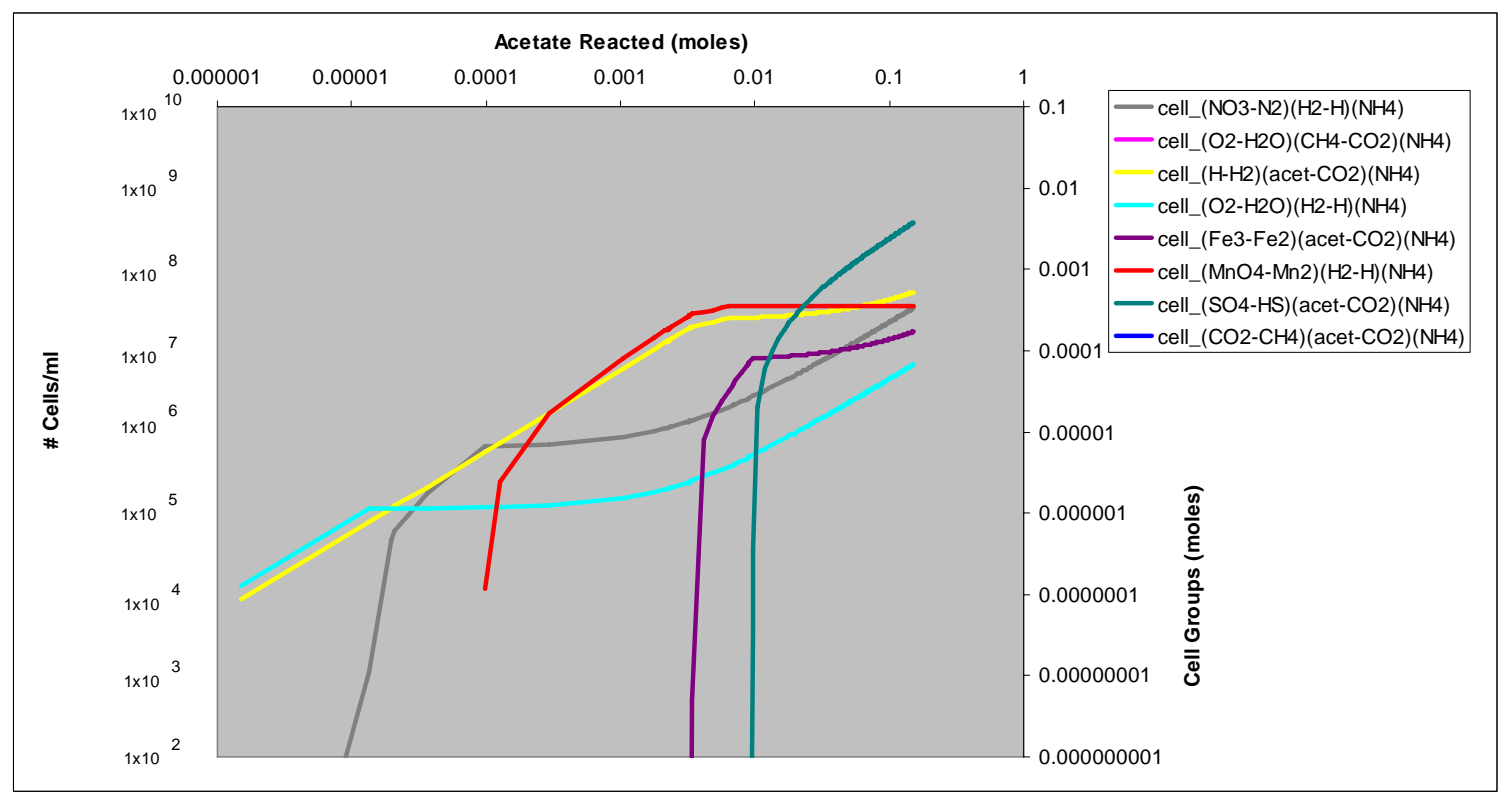

